TITLE:

\title{
Solvation effects in near-critical binary mixtures
}

$\operatorname{AUTHOR}(S)$ :

Onuki, A; Kitamura, $\mathrm{H}$

\section{CITATION:}

Onuki, A ... [et al]. Solvation effects in near-critical binary mixtures. JOURNAL OF CHEMICAL PHYSICS 2004, 121(7): 3143-3151

ISSUE DATE:

2004-08-15

URL:

http://hdl.handle.net/2433/49911

\section{RIGHT:}

Copyright 2004 American Institute of Physics. This article may be downloaded for personal use only. Any other use requires prior permission of the author and the American Institute of Physics. 


\title{
Solvation effects in near-critical binary mixtures
}

\author{
Akira Onuki and Hikaru Kitamura \\ Department of Physics, Kyoto University, Kyoto 606-8502, Japan
}

(Received 8 April 2004; accepted 14 May 2004)

\begin{abstract}
A Ginzburg-Landau theory is presented to investigate solvation effects in near-critical polar fluid binary mixtures. Concentration dependence of the dielectric constant gives rise to a shell region around a charged particle within which solvation occurs preferentially. As the critical point is approached, the concentration has a long-range Ornstein-Zernike tail representing strong critical electrostriction. If salt is added, strong coupling arises among the critical fluctuations and the ions. The structure factors of the critical fluctuations and the charge density are calculated and the phase transition behavior is discussed. (C) 2004 American Institute of Physics. [DOI: 10.1063/1.1769357]
\end{abstract}

\section{INTRODUCTION}

Solvation effects are of great importance in understanding the degree of solubility of ions in various polar solvents. ${ }^{1,2}$ A large number of papers have been devoted on this problem. ${ }^{3-6}$ The theoretical approaches range from solving phenomenological continuum models to performing computer simulations on microscopic models. Originally, Born used a simple continuum theory of electrostatics to derive the solvation (polarization) free energy of a single ion with charge $Z e,{ }^{1,7}$

$$
\Delta G_{\mathrm{B}}=-\left(Z^{2} e^{2} / 2 R_{\mathrm{B}}\right)(1-1 / \varepsilon),
$$

where $R_{\mathrm{B}}$ represents the ionic radius. ${ }^{1,8}$ In his theory the solvent dielectric constant $\varepsilon$ is assumed to be homogeneous and the contribution without polarization $(\varepsilon=1)$ or in vacuum is subtracted. In the line of continuum models, considerable efforts have been made to take into account the dielectric saturation near the ion core (which are particularly crucial for multivalent ions). ${ }^{9,10}$ The Born theory also neglects possible inhomogeneity of $\varepsilon$ in the vicinity of the ion due to a change in the density for one-component fluids or in the concentration for binary fluid mixtures (electrostriction). ${ }^{11-14}$

We point out that the previous theories have not yet treated the physics on mesoscopic scales such as solvation effects on phase transitions or collective phenomena at nondilute salt concentrations. In this paper we will show that such effects can be conveniently studied within a scheme of Ginzburg-Landau theory. First, we will apply this approach to solvation around a charged particle in polar binary mixtures near the consolute critical point. Here important is the gradient free energy well known in the field of critical phenomena ${ }^{15}$ which will reasonably accounts for the longrange electrostriction around ions. In the previous theories ${ }^{11-13}$ in the physical chemistry, the gradient free energy has been neglected. On the basis of these results we will then discuss the effects of low-density salt in polar binary mixtures in a Debye-Hückel-type approximation. This second part is an extension of a previous paper by one of the present authors. ${ }^{16}$ We also note that similar effects have re- cently been discussed when the solvent is a liquid crystal, ${ }^{17}$ where the director field can be much deformed around a charged particle over a long distance.

\section{SOLVATION AROUND A SINGLE CHARGED PARTICLE}

\section{A. Electrostatics}

We place a charged particle in a near-critical binary mixture in a one-phase state in equilibrium, in which the dielectric constant $\varepsilon=\varepsilon(\phi)$ depends strongly on the concentration. This is the case when the two components have very different dielectric constants, $\varepsilon_{A}$ and $\varepsilon_{B}$, with $\varepsilon_{A}$ considerably larger than $\varepsilon_{B}$. There is no established theory of the dielectric constant of such mixtures. However, empirically, $\varepsilon$ can be expressed roughly as a linear function of the concentration in many relevant mixtures. ${ }^{2}$ In the present theory, for simplicity, we assume the linear dependence,

$$
\varepsilon=\varepsilon_{0}+\varepsilon_{1} \phi
$$

where $\varepsilon_{0}$ and $\varepsilon_{1}$ are constants (with $\varepsilon_{B}=\varepsilon_{0}$ and $\varepsilon_{A}=\varepsilon_{0}$ $\left.+\varepsilon_{1}\right)$ and $\phi$ is the concentration or the volume fraction of the preferred component $A$. Debye and Kleboth ${ }^{18}$ performed a light scattering experiment on a mixture of nitrobenzene (NB) + 2,2,4-trimethylpentane in electric field, for which $\varepsilon(\phi)$ increased from 2.1 to 34.2 with increasing NB with $\partial^{2} \varepsilon / \partial \phi^{2}=28.7$ near the critical point. The measured curve of $\varepsilon(\phi)$ versus $\phi$ may be fairly fitted to the linear form (2.1) (and fitting is better in the region $\phi \geq \phi_{c}$ ). In their experiment the critical temperature was $29.16^{\circ} \mathrm{C}$ and the critical concentration was $51.7 \mathrm{wt} \%$ of NB (corresponding to a volume fraction of 0.381 of NB). In this paper the dielectric constant is assumed to be independent of electric field, although this is a questionable assumption in the vicinity of small ions. ${ }^{9,10}$

The electric field $\mathbf{E}=-\boldsymbol{\nabla} \Phi$ is induced by the electric charges and the electric potential $\Phi$ satisfies

$$
\boldsymbol{\nabla} \cdot \varepsilon \boldsymbol{\nabla} \Phi=-4 \pi \rho(\mathbf{r}),
$$

where $\rho(\mathbf{r})$ is the charge density. In our problem the space dependence of $\varepsilon$ is crucial. 


\section{B. Ginzburg-Landau free energy}

We construct a simple theory for a nearly incompressible mixture near the consolute critical point at a given pressure. Then $\psi=\phi-\phi_{\mathrm{c}}$ is the order parameter of liquid-liquid phase transition, where $\phi_{\mathrm{c}}$ is the critical concentration. The total Ginzburg-Landau free energy of the system is given by

$$
F=\int d \mathbf{r}\left[f+\frac{C}{2}|\nabla \phi|^{2}+\frac{\varepsilon}{8 \pi} \mathbf{E}^{2}\right] .
$$

The molecules of the two components have a common volume $v_{0}=a^{3}$, although they can have different volumes in general. The free-energy density $f=f(\phi)$ is given by the Hildebrand ${ }^{19}$ (or Bragg-Williams ${ }^{15}$ ) expression

$$
f=\frac{k_{\mathrm{B}} T}{v_{0}}[\phi \ln \phi+(1-\phi) \ln (1-\phi)+\chi \phi(1-\phi)],
$$

where $\chi$ is the interaction parameter dependent on the temperature $T$. The critical-point values of $\phi$ and $\chi$ are

$$
\phi_{\mathrm{c}}=1 / 2, \quad \chi_{\mathrm{c}}=2,
$$

respectively, in the mean field theory. The order parameter is defined by

$$
\psi=\phi-1 / 2 .
$$

The coefficient $C$ of the gradient term is of order $k_{\mathrm{B}} T / a$ and we will use a simple form ${ }^{20,21}$

$$
C=k_{\mathrm{B}} T \chi / a \text {. }
$$

The last term in $F$ is the electrostatic contribution. The freeenergy functional (2.3) supplemented with Eq. (2.2) can be used generally in the presence of ions in polar near-critical fluids.

\section{Preferential solvation}

We consider a single charged particle at the origin of the reference frame. The charged particle has a radius $R$. It is convenient to assume that the charge density is homogeneous within the sphere as

$$
\rho=Z e /\left(4 \pi R^{3} / 3\right) \quad(r<R)
$$

and vanishes outside the sphere $r>R$. The total charge is given by $Z e$. In our theory $R$ is a phenomenological parameter. Then the volume fraction $\phi$ and the electric potential $\Phi$ can be defined even within the sphere and, for the single charge case, all the quantities may be assumed to depend only on space as

$$
\phi=\phi(r), \quad \Phi=\Phi(r), \quad \mathbf{E}=-\Phi^{\prime}(r) r^{-1} \mathbf{r},
$$

where $r=|\mathbf{r}|$ and $\Phi^{\prime}=d \Phi / d r$. The concentration $\phi(r)$ decrease from $\phi(0)$ to $\phi_{\infty}$ with increasing $r$ (see Fig. 1 to be presented below). In this case Eq. (2.2) is solved to give

$$
-\varepsilon(\phi) \Phi^{\prime}(r)=\frac{4 \pi}{r^{2}} \int_{0}^{r} d r_{1} r_{1}^{2} \rho\left(r_{1}\right)=\frac{Z e}{r^{2}} \theta(r),
$$

where $\theta(r)=1$ for $r>R$ and

$$
\theta(r)=(r / R)^{3} \quad(r<R)
$$

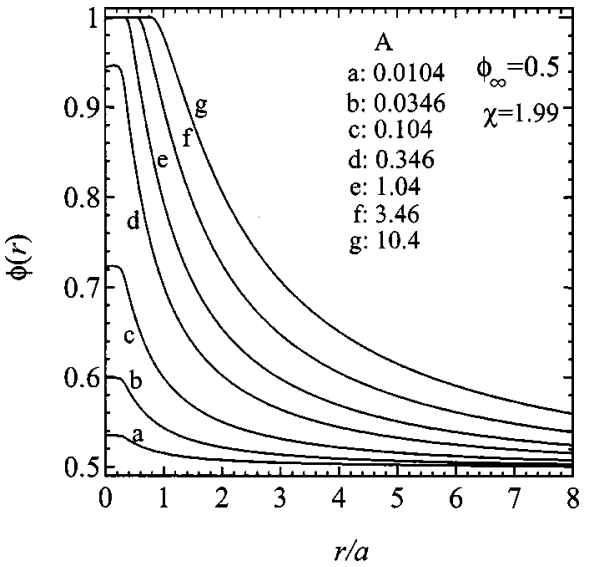

FIG. 1. Concentration profile $\phi(r)$ around an ion for various $A$ at $\chi$ $=1.99$ where $\xi=10 a$ and $\phi \rightarrow \phi_{\infty}=1 / 2$ far from the ion.

under the assumption (2.8). The electrostatic free energy is now simply of the form

$$
\int d \mathbf{r} \frac{1}{8 \pi} \varepsilon \mathbf{E}^{2}=\frac{1}{2} Z^{2} e^{2} \int_{0}^{\infty} d r \frac{1}{\varepsilon r^{2}} \theta(r)^{2} .
$$

If $\varepsilon$ is replaced by the dielectric constant far from the ion $\varepsilon_{\infty}$, the above quantity is integrated to become a Born contribution [see Eq. (1.1)],

$$
F_{\mathrm{B} \infty}=Z^{2} e^{2} / 2 \varepsilon_{\infty} R_{\mathrm{B}} .
$$

In terms of the critical-point dielectric constant $\varepsilon_{\mathrm{c}}=\varepsilon_{0}$ $+\varepsilon_{1} / 2$ and the order parameter $\psi_{\infty}=\phi_{\infty}-1 / 2$ far from the ion, we have

$$
\varepsilon_{\infty}=\varepsilon_{0}+\varepsilon_{1} \phi_{\infty}=\varepsilon_{\mathrm{c}}+\varepsilon_{1} \psi_{\infty} .
$$

The Born radius is given by

$$
R_{\mathrm{B}}=5 R / 6 .
$$

In the integral (2.12) the contribution from the ion interior $r<R$ is $1 / 5$ of that from the ion exterior, resulting in the factor $1 /(1+1 / 5)=5 / 6$ in Eq. (2.15). However, if we replace $\varepsilon$ by its value at the ion $\varepsilon(0)=\varepsilon_{0}+\varepsilon_{1} \phi(0)$, we obtain another estimate,

$$
F_{\mathrm{B} 0}=Z^{2} e^{2} / 2 \varepsilon(0) R_{\mathrm{B}}=F_{\mathrm{B} \infty} \varepsilon_{c} /\left[\varepsilon_{0}+\varepsilon_{1} \phi(0)\right] .
$$

As will be calculated in the Appendix, the equilibrium free energy of a single ion accounting for the inhomogeneous $\phi$ and $\varepsilon$ in the mean field theory reads

$$
\begin{aligned}
F_{\text {sol }} & =4 \pi \int_{0}^{\infty} d r r^{2}\left[\hat{f}+C\left(\phi^{\prime}\right)^{2} / 2+\varepsilon \mathbf{E}^{2} / 8 \pi\right] \\
& =\frac{4 \pi}{3} \int_{0}^{\infty} d r\left[C r^{2}\left(\phi^{\prime}\right)^{2}+\frac{Z^{2} e^{2} \theta^{2}}{8 \pi r^{4}} \frac{d}{d r}\left(\frac{r^{3}}{\varepsilon}\right)\right],
\end{aligned}
$$

where

$$
\hat{f}(\phi)=f(\phi)-f\left(\phi_{\infty}\right)-\mu\left(\phi-\phi_{\infty}\right),
$$

with $\mu=f^{\prime}\left(\phi_{\infty}\right)$ being the chemical potential difference. As shown in the second line of Eq. (2.17), $F_{\text {sol }}$ consists of the gradient contribution $(\propto C)$ and the electrostatic contribution 
$\left(\propto e^{2}\right)$. The latter becomes the Born contribution (2.13) or (2.16) if $\varepsilon$ is replaced by $\varepsilon_{\infty}$ or $\varepsilon(0)$. In Fig. 7 below we shall see the relation $F_{\mathrm{B} 0}<F_{\text {sol }}<F_{\mathrm{B} \infty}$.

With the aid of Eq. (2.11) the minimization condition $\delta F / \delta \phi=\mu=$ const in equilibrium is rewritten as

$$
\ln \left[\frac{1+2 \psi}{1-2 \psi}\right]-2 \chi \psi-\chi a^{2} \nabla^{2} \psi-\nu=A \frac{a^{4} \theta^{2}}{\hat{\varepsilon}^{2} r^{4}},
$$

where $\hat{\varepsilon}$ is the normalized dielectric constant,

$$
\hat{\varepsilon}=\varepsilon / \varepsilon_{\mathrm{c}}=1+\left(\varepsilon_{1} / \varepsilon_{\mathrm{c}}\right) \psi .
$$

The dimensionless chemical potential difference $\nu$ $=v_{0} \mu / k_{\mathrm{B}} T$ is written in terms of $\psi_{\infty}=\psi(\infty)$ as

$$
\nu=\ln \left[\left(1+2 \psi_{\infty}\right) /\left(1-2 \psi_{\infty}\right)\right]-2 \chi \psi_{\infty} .
$$

The coefficient $A$ on the right-hand side of Eq. (2.19) represents the strength of solvation defined by

$$
A=\varepsilon_{1} Z^{2} e^{2} / 8 \pi \varepsilon_{\mathrm{c}}^{2} k_{\mathrm{B}} T a=\varepsilon_{1} Z^{2} \ell_{\mathrm{B}} / 8 \pi \varepsilon_{\mathrm{c}} a .
$$

In the second line the Bjerrum length

$$
\ell_{\mathrm{B}}=e^{2} / \varepsilon_{\mathrm{c}} k_{\mathrm{B}} T
$$

at the critical concentration is introduced. For example, if we set $\ell_{\mathrm{B}} \sim 14 \AA, a \sim 2 \AA, \varepsilon_{1} / \varepsilon_{0} \sim 2$, we obtain $A \sim Z^{2} / 5$. In the vicinity of the ion the right-hand side of Eq. (2.19) takes a maximum of order $A a^{4} / R^{4}$ (because $\hat{\varepsilon} \sim 1$ ). If this maximum is much larger than 1 , there arises a solvation shell around the ion within which preferential solvation is strong and $\phi$ $\cong 1$. Thus the condition of strong solvation may be expressed as

$$
A>(R / a)^{4} .
$$

The solvation is stronger for smaller $R / a$, which is a wellknown result in the literature. ${ }^{1}$

It is worth noting that Padova presented an equation similar to Eq. (2.19) for the concentration in the vicinity of an ion using thermodynamic arguments, ${ }^{11}$ where $C=0$ and the gradient term $\propto \nabla^{2} \Psi$ did not appear $(C=0)$. Even far from the critical point, as can be seen in Fig. 8 below, the result with $C=0$ is considerably different from that with the gradient term in the strong solvation case.

\section{Relations near the critical point}

If the system is near the critical point far from the charged particle, we have $\chi \cong 2$ and $\phi_{\infty} \cong 1 / 2$. Then the inverse correlation length $\kappa=\xi^{-1}$ far from the ion is determined by

$$
a^{2} \kappa^{2}=4 /\left(1-4 \psi_{\infty}^{2}\right) \chi-2 \cong 2-\chi+8 \psi_{\infty}^{2} .
$$

As $T \rightarrow T_{\mathrm{c}}$ in the upper critical solution temperature (UCST) case we may set

$$
2-\chi \cong D_{1}\left(T / T_{\mathrm{c}}-1\right) .
$$

The correlation length in the mean field expression becomes $\xi=\xi_{0+}\left(T / T_{\mathrm{c}}-1\right)^{-1 / 2}$ with

$$
\xi_{0+}=a D_{1}^{-1 / 2}
$$

in one-phase states at the critical composition. ${ }^{18}$ The fluid can remain near-critical only in the region where $A a^{4} / r^{4}$ $\ll 1$ or

$$
r \gg A^{1 / 4} a .
$$

The shell radius should thus grow as $A^{1 / 4} a$ for $A \gg 1$. In the near-critical region, Eq. (2.19) may be approximated in the Landau expansion form, ${ }^{16}$

$$
a^{2}\left[\kappa^{2}-\nabla^{2}\right] \delta \psi+\frac{8}{3}\left(3 \psi_{\infty} \delta \psi^{2}+\delta \psi^{3}\right)=A \frac{a^{4} \theta^{2}}{2 r^{4}},
$$

where $\delta \psi=\psi-\psi_{\infty}$. This equation does not hold within the solvation shell if $\psi$ is not small there for strong solvation, but holds generally far from the ion. If we retain the linear terms only in the above equation, we obtain $\left(\kappa^{2}-\nabla^{2}\right) \delta \psi \cong 0$ far from the ion, so there should emerge the Ornstein-Zernike tail,

$$
\delta \psi(r) \cong \frac{B}{r} e^{-\kappa r},
$$

in the concentration. The coefficient $B$ is a microscopic length to be determined in the following.

In the Landau theory of phase transition (usually presented in the context of Ising spin systems), ${ }^{15}$ the right-hand side of Eq. (2.19) or Eq. (2.29) plays the role of a (positiondependent) magnetic field $h$. If $h$ were homogeneous and small, we would have the linear response $\delta \psi \sim h / \kappa^{2}$. In the present case, however, $h=h(r)$ steeply changes in space $\left(\propto r^{-4}\right)$ from a maximum of order $A a^{4} / R^{4}$ to zero and as a result the gradient term $-\nabla^{2} \psi$ becomes increasingly crucial with decreasing $\kappa$.

\section{E. Weak solvation limit near the critical point}

It is instructive first to consider the simplest case of very small $A$ near the critical point where $0<2-\chi \ll 1$ and $\psi_{\infty}$ $=0$. Furthermore, if $\psi(0) \ll \kappa a$ holds at the ion, we may neglect the second term on the left-hand side of Eq. (2.29) in the whole space to obtain the linearized equation,

$$
\left(\kappa^{2}-\nabla^{2}\right) \psi=A \theta(r)^{2} a^{2} / 2 r^{4},
$$

where we have set $\hat{\varepsilon}=1$. This equation is readily integrated to give

$$
\psi(r)=\frac{A a^{2}}{4 \kappa r} \int_{0}^{\infty} \frac{d r_{1}}{r_{1}^{3}} e^{-\kappa\left|r-r_{1}\right|} \theta\left(r_{1}\right)^{2}-\frac{D}{r} e^{-\kappa r},
$$

where the coefficient $D$ is determined such that $\psi(0)$ is finite. We can see the relations

$$
\begin{aligned}
\psi(0)=2 \kappa D & =A a^{2} \int_{0}^{\infty} d r_{1} e^{-\kappa r_{1}} \theta\left(r_{1}\right)^{2} / 2 r_{1}^{3} \\
& \cong 3 A a^{2} / 8 R^{2} \quad(\kappa R \ll 1) .
\end{aligned}
$$

The second line holds for $\kappa R \ll 1$. As stated above (2.31), the linearization is allowed for

$$
A \ll \kappa R^{2} / a \quad \text { or } \quad \varepsilon_{1} Z^{2} \ell_{\mathrm{B}} / 8 \pi \varepsilon_{\mathrm{c}} \ll R^{2} \kappa .
$$

From Eq. (2.31) the three-dimensional Fourier transformation of $\psi$ becomes $\psi_{\mathbf{k}}=H_{k} /\left(\kappa^{2}+k^{2}\right)$, where

$$
H_{k}=2 \pi A a^{2} \int_{0}^{\infty} d r \theta(r)^{2} \sin (k r) / k r^{3} \text {. }
$$


Here $H_{k}$ tends to a well-defined long-wavelength limit $H_{0}$ $=\lim _{k \rightarrow 0} H_{k}$ for $k R \ll 1$. Thus, for $\kappa R \ll 1, \psi(r)$ approximately takes the Ornstein-Zernike form (2.30) in a wide space region with $B=a^{2} H_{0} / 4 \pi$. Here,

$$
B=A a^{2} \int_{0}^{\infty} d r \theta(r)^{2} / 2 r^{2}=A a^{2} / 2 R_{\mathrm{B}},
$$

with $R_{\mathrm{B}}$ being given by Eq. (2.15). This form also directly follows from the integral form (2.32) (by taking the limit $\kappa$ $\rightarrow 0$ ). If we do not assume the special form $C$ in Eq. (2.7), we have $B=\left(k_{\mathrm{B}} T / C\right) A a / R_{\mathrm{B}}$ [which will be used in Eq. (3.12) below].

At very long distances $r>\lambda$, however, the gradient term $-\nabla^{2} \psi$ in Eq. (2.31) becomes negligible to give

$$
\psi(r) \cong A a^{2} / 2 \kappa^{2} r^{4} \text {. }
$$

This is the relation of linear response as stated at the end of Sec. III D. The crossover length $\lambda$ is longer than $\xi=\kappa^{-1}$ and is determined by the balance of the Ornstein-Zernike form (2.30) and the long-distance limit (2.37) as

$$
(\kappa \lambda)^{3} e^{-\kappa \lambda}=\kappa R .
$$

For example, $\lambda \cong 10.8 \xi$ for $R=0.03 \xi$. Thus $\kappa \lambda$ increases as $\kappa R$ decreases.

We may then calculate the excess accumulation $\Psi$ of the component $A$ around the ion as

$$
\Psi=\int d \mathbf{r} \psi(r)=2 \pi A a^{2} / \kappa^{2} R_{\mathrm{B}}=4 \pi B / \kappa^{2},
$$

which coincides with the space integral of the OrnsteinZernike form (2.30) and grows strongly on approaching the critical point. The integral of the tail (2.38) in the region $r$ $>\lambda$ is much smaller than the value in Eq. (2.39) by $R / \lambda$ and is canceled with a contribution from the correction to the Ornstein-Zernike form in the range $r<\lambda$.

\section{F. Behavior at the critical point}

At the critical point ( $\chi=2$ and $\left.\phi_{\infty}=0.5\right), \psi(r)$ is of the form $\mathrm{Ba} / \mathrm{r}$ at long distances in the linear theory. We here show that it is delicately modified in the nonlinear theory. We rewrite Eq. (2.29) for $r \gg a$ as

$$
-a^{2} \nabla^{2} \psi+\frac{8}{3} \psi^{3}=A \frac{a^{4}}{2 r^{4}} .
$$

We set $G(s)=r \psi(r) / a$ with $s=\ln (1+r / a)$ to obtain

$$
\left(1-e^{-s}\right)^{2}\left[\frac{d^{2}}{d s^{2}}-\frac{d}{d s}\right] G(s)=\frac{8}{3} G(s)^{3}-\frac{1}{2} A e^{-s} .
$$

For $s \gg 1$ we assume the algebraic decay $G(s) \sim s^{-\beta}$. Then the above equation is approximated as $-d G(s) / d s$ $\cong 8 G(s)^{3} / 3$, yielding the solution

$$
G(s) \cong\left(3^{1 / 2} / 4\right) s^{-1 / 2}
$$

with $\beta=1 / 2$. Thus the asymptotic behavior at extremely long distances $s \gg 1$ should be

$$
\psi(r) \cong 3^{1 / 2} a /[4 r \sqrt{\ln (1+r / a}) .
$$

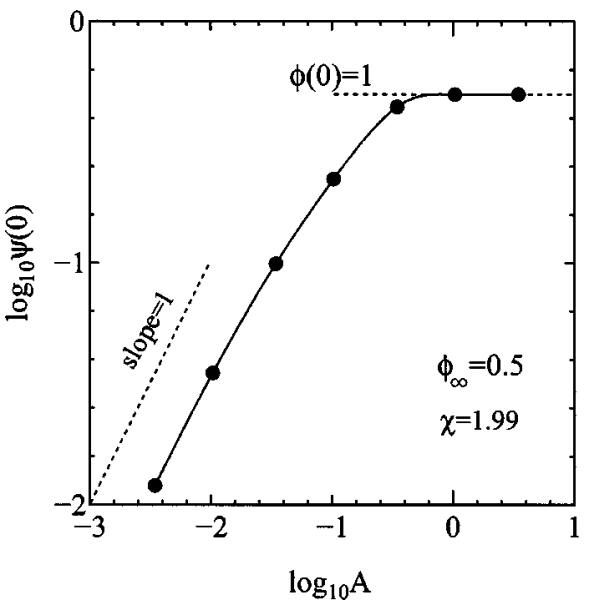

FIG. 2. Concentration deviation $\psi(0)=\phi(0)-1 / 2$ at the ion center for various $A$ at $\chi=1.99$.

The above form will turn out to be consistent with numerical analysis in Fig. 6 to be presented below.

\section{G. Numerical results}

We then show numerical solutions of Eq. (2.19) around an ion in equilibrium one-phase states for the case $\varepsilon_{0}=10$ and $\varepsilon_{1}=70$ in Eq. (2.1), assuming the model charge distribution (2.8). The ion radius is given by $R=0.3 a$ in terms of the solvent radius $a$.

We show the concentration profile, $\phi(r)$ versus $r / a$, in Fig. 1 and the concentration $\phi(0)$ at the ion center in Fig. 2. Notice that $\phi(r) \cong \phi(0)$ within the interior of the ion $r \lesssim R$ owing to the smoothing effect of the gradient term. We can see that the concentration $\phi(0)$ at the ion increases obeying the linear growth (2.31) for $A \ll 1$ and saturates into 1 for $A \gtrsim 0.3$ and that the shell region expands with increasing $A$. The shell radius $R_{\text {shell }}$ may be defined such that $\phi(r)>0.75$ for $r<R_{\text {shell }}$, say. Then $R_{\text {shell }} \sim A^{\alpha}$ with the effective exponent $\alpha$ being about $1 / 4$ for the profiles (d) $-(\mathrm{g})$ in Fig. 1 . Below (2.28) we have discussed that $\alpha$ should tend to $1 / 4$ for $A \gg 1$.

In Fig. 3 we examine the behavior of $\psi(r)=\phi(r)-1 / 2$ with $\phi_{\infty}=1 / 2$ at $A=0.346$. We can see that the combination $r \psi(r) / a$ behaves as $(B / a) \exp (-\kappa r)$ in a wide intermediate range $a \leq r \leq \lambda$. The correlation length $\xi=\kappa^{-1}$ is given by $10 a$ for $\chi=1.99$ and by $10^{3 / 2} a$ for $\chi=1.999$. For $r \gtrsim \lambda, \psi(r)$ can be fitted to the $r^{-4}$ tail in Eq. (2.37). Figure 4 displays $B / a$ versus $A$ for $\chi=1.99$ and 1.999 at $\phi_{\infty}=0.5$. In the nonlinear regime $A \gtrsim 0.1$ the data may be fitted to $B / a \sim A^{0.4}$. In Fig. 5 we plot the crossover length $\lambda$ divided by $a$ obtained from equating the two fitted functions (2.30) and (2.37). In the linear regime with small $A, \lambda$ is given by Eq. (2.37).

The concentration profile just at the critical point is also of great interest. In Fig. 6 we plot the combination $\psi(r) r s^{1 / 2}$ versus $s=\ln (1+r / a)$ in the region $s<12$ or $r / a<e^{12}-1$ for $A=0.2,1$, and 5. If Eq. (2.42) is valid, this combination should tend to $3^{1 / 2} / 4 \cong 0.43$. Thus the three curves are consistent with Eq. (2.42).

Figure 7 displays the numerically calculated solvation free energy $F_{\text {sol }}$ in Eq. (2.17) divided by $F_{\text {Bo }}$ in Eq. (2.13). 


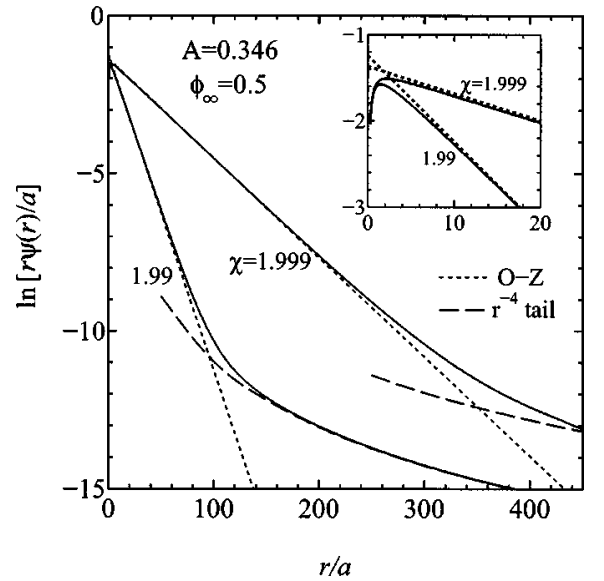

FIG. 3. Numerical $r \psi(r) / a$ vs $r / a$ on a semilogarithmic scale at $A$ $=0.346$ for $\chi=1.99$ (lower solid line) and 1.999 (upper solid line) with $\phi_{\infty}=1 / 2$. The numerical $\psi(r)=\phi(r)-1 / 2$ can be excellently fitted to the Ornstein-Zernike form (corresponding to dotted lines) in the intermediate range $a \lesssim r \lesssim \lambda$, while it can be fitted to the $r^{-4}$ tail (2.37) (corresponding to broken lines) for $r \geq \lambda$. The curve of $\lambda$ vs $A$ will be given in Fig. 5 .

Also plotted is the ratio $F_{\mathrm{B} 0} / F_{\mathrm{B} \infty}=\varepsilon_{c} /\left[\varepsilon_{0}+\varepsilon_{1} \phi(0)\right]$ in Eq. (2.26). Thus we find that $F_{\text {sol }}$ is intermediate between $F_{\mathrm{B} 0}$ and $F_{\mathrm{B} \infty}$. Coincidence of the two curves of $\chi=1.99$ and 1.999 implies that $F_{\text {sol }}$ tends to a constant on approaching the critical point.

In Fig. 8 we demonstrate that $r \psi(r) / a$ versus $r / a$ far above the critical point with $\chi=1.5$ and $\phi_{\infty}=0.5$. The profile can be fitted neither to the Ornstein-Zernike form nor to the solution of Eq. (2.19) without the gradient term. The curves from the local equilibrium theory without the gradient term ${ }^{11}$ considerably differ from that in our theory even away from the critical point.

\section{NEAR-CRITICAL FLUIDS WITH IONS}

Experimentally, it has long been known that even a small fraction of ions (salt) with $c \ll 1$ dramatically changes the

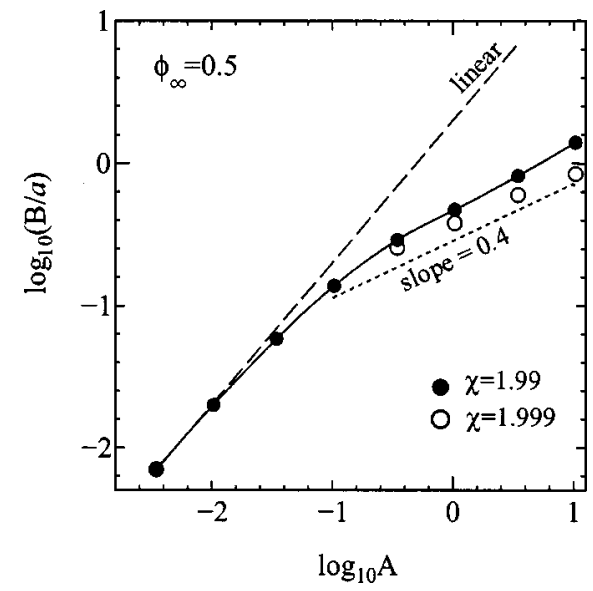

FIG. 4. Coefficient $B$ of the Ornstein-Zernike tail in the concentration profile as a function of $A$ at $\phi_{\infty}=0.5$ for $\chi=1.99$ and 1.999. It exhibits only weak dependence on $2-\chi \propto T-T_{\mathrm{c}}$.

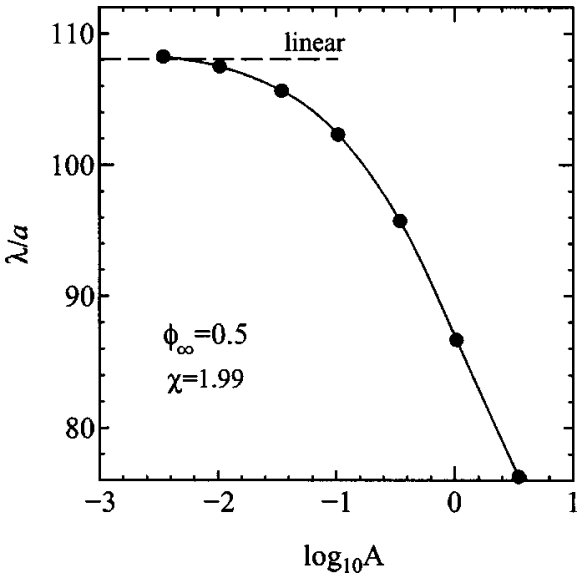

FIG. 5. Crossover length $\lambda$ from the Ornstein-Zernike tail (2.30) to the $r^{-4}$ tail (2.37) at $\phi_{\infty}=0.5$ for $\chi=1.99$ where $\xi=10 a$.

liquid-liquid phase behavior, where $c$ is the mass or mole fraction of ions. For small $c$, the UCST coexistence curve shifts upward as

$$
\Delta T_{\text {ion }}=A_{\text {ion }} c+O\left(c^{2}\right),
$$

with large positive coefficient $A_{\text {ion }}$, expanding the region of demixing. For example, $A_{\text {ion }} / T_{\mathrm{c}} \sim 10$ with $T_{\mathrm{c}} \sim 300 \mathrm{~K}$ when $\mathrm{NaCl}$ was added to cyclohexane + methyl alcohol ${ }^{22}$ and to triethylamine $+\mathrm{H}_{2} \mathrm{O}^{23}$ The LCST coexistence curve of 2,6-lutidine $+\mathrm{H}_{2} \mathrm{O}\left(\mathrm{D}_{2} \mathrm{O}\right)$, the shift is downward with $\left|A_{\text {ion }}\right| / T_{\mathrm{c}} \sim 10 .^{24}$ Similar large impurity effects were observed when water was added to methanol+cyclohexane. ${ }^{25}$ In some aqueous mixtures, even if they are miscible at all $T$ at atmospheric pressure without salt, addition of a small amount of salt gives rise to reentrant phase separation behavior. ${ }^{26-29}$ Such reentrant phase behavior is believed to arise from hydrogen bonding.

\section{A. Ginzburg-Landau free energy}

In the preceding section we have examined the concentration profile around a single fixed charged particle. We then need to construct a theory on polar binary mixtures in which a small amount of salt is doped. ${ }^{16}$ Even if the ion concentration is very small, the interactions among the ions should be

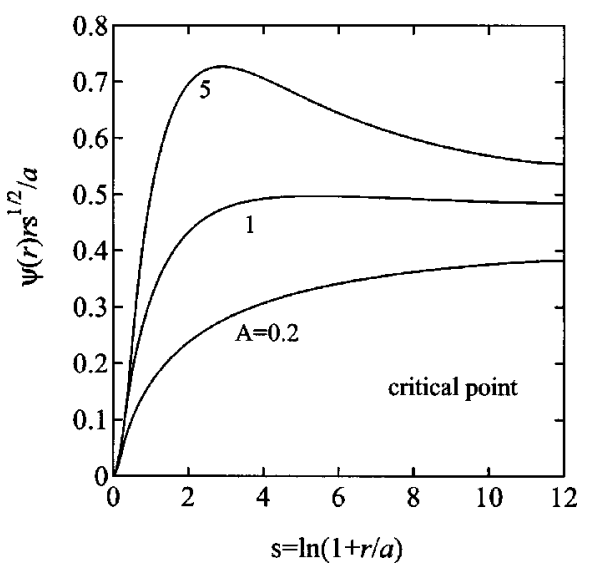

FIG. 6. $\psi(r) r s^{1 / 2}$ vs $s=\ln (1+r / a)$ at the critical point for $A=0.2,1$, and 5 . The curves appear to approach $\sqrt{3} / 4$ very slowly supporting (2.43). 


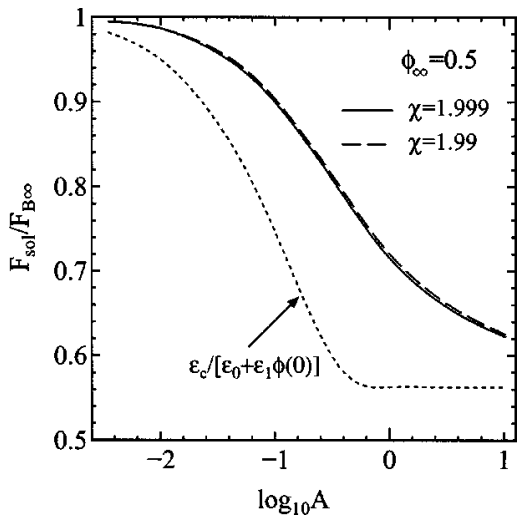

FIG. 7. Solvation free energy $F_{\text {sol }}$ in Eq. (2.17) divided by $F_{\mathrm{B} \infty}$ in Eq. (2.13) as a function of $A$. The two curves for $\chi=1.99$ (broken line) and 1.999 (solid line) are almost identical. The dotted line represents $F_{\mathrm{B} 0} / F_{\mathrm{B} \infty}$ at $\chi$ $=1.99$ [see Eq. (2.16)].

much influenced by the preferential solvation near the critical point. We here present a Ginzburg-Landau free energy in which the fluctuations on the spatial scale of the solvation shell radius $R_{\text {shell }}$ have been coarse grained. Then the order parameter $\psi(\mathbf{r})=\phi(\mathbf{r})-\phi_{\mathrm{c}}$ and the ion densities $n_{1}(\mathbf{r})$ and $n_{2}(\mathbf{r})$ for the two species do not change appreciably on the scale of $R_{\text {shell }}$.

The two ion species, 1 and 2, have charges, $Q_{1}=Z e$ and $Q_{2}=-e$. The average densities are written as

$$
\left\langle n_{1}\right\rangle=\bar{n}, \quad\left\langle n_{2}\right\rangle=Z \bar{n},
$$

where $\langle\cdots\rangle$ denotes taking the space average. Notice that the charge neutrality condition yields $Z\left\langle n_{1}\right\rangle=\left\langle n_{2}\right\rangle$. The total charge number density is written as

$$
n_{\text {tot }}=(Z+1) \bar{n}
$$

We propose the free energy $F$ in the form ${ }^{16}$

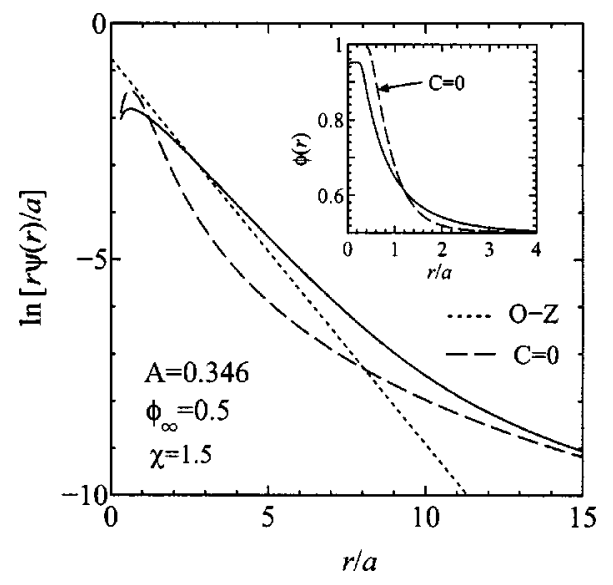

FIG. 8. $r \psi(r)$ vs $r / a$ on a semilogarithmic scale around an ion far above the critical point with $\chi=1.5$ and $\phi_{\infty}=0.5$. The profile can be fitted neither to the Ornstein-Zernike form (dotted line) nor to the local equilibrium curve without the gradient term (broken line). The inset displays $\phi(r)$ vs $r / a$ near the ion on a linear scale.

$$
\begin{aligned}
F= & \int d \mathbf{r}\left[f(\psi)+\frac{C}{2}|\nabla \psi|^{2}+\frac{\varepsilon_{\mathrm{c}}}{8 \pi} \mathbf{E}^{2}\right. \\
& \left.+k_{\mathrm{B}} T \sum_{K=1,2}\left(n_{K} \ln n_{K}+w_{K} n_{K} \psi\right)\right] .
\end{aligned}
$$

The first line is of the same form as the free energy in Eq. (2.3), but $f(\phi)$ can be expressed in the Landau expansion form $f=r \psi^{2} / 2+u_{0} \psi^{4} / 4$ because the noncritical behavior in the solvation shell is not treated here. In addition we do not assume the special form (2.7) for the coefficient $C$. The electric field $\mathbf{E}=-\boldsymbol{\nabla} \Phi$ here arises from the Coulomb interaction among the ions so that

$$
\nabla \cdot \mathbf{E}=-\nabla^{2} \Phi=4 \pi \varepsilon_{\mathrm{c}}^{-1} \rho(\mathbf{r}),
$$

where

$$
\rho(\mathbf{r})=e\left[Z n_{1}(\mathbf{r})-n_{2}(\mathbf{r})\right]
$$

is the charge density. Therefore, the electrostatic part of the free energy is rewritten as

$$
\int d \mathbf{r} \frac{\varepsilon_{\mathrm{c}}}{8 \pi} \mathbf{E}^{2}=\frac{1}{2 \varepsilon_{\mathrm{c}}} \int d \mathbf{r} \int d \mathbf{r}^{\prime} \rho(\mathbf{r}) \rho\left(\mathbf{r}^{\prime}\right) \frac{1}{\left|\mathbf{r}-\mathbf{r}^{\prime}\right|} .
$$

Because the small-scale electrostriction on the scale of $R_{\text {shell }}$ has been coarse grained, we neglect the small-scale inhomogeneity of the dielectric constant and set $\varepsilon=\varepsilon_{\mathrm{c}}$ with $\varepsilon_{\mathrm{c}}$ being the dielectric constant at the critical point.

The free energy with the bilinear coupling $\left(\propto n_{K} \psi\right)$ was already examined in Refs. 30 and 31, where the coupling constants $w_{K}$ were phenomenological parameters, however. We claim that the small-scale solvation or electrostriction is the origin of the bilinear coupling. In fact, the minimization of $F$ at fixed inhomogeneous ion densities yields

$$
\mu=f^{\prime}-C \nabla^{2} \psi+k_{\mathrm{B}} T\left(w_{1} n_{1}+w_{2} n_{2}\right)=\mathrm{const},
$$

where $f^{\prime}=\partial f / \partial \psi$. For small inhomogeneous deviations $\delta \psi$ $=\psi-\langle\psi\rangle$ and $\delta n_{K}=n_{K}-\left\langle n_{K}\right\rangle(K=1,2)$ we linearize the above equation to obtain

$$
C\left(\kappa^{2}-\nabla^{2}\right) \delta \psi+k_{\mathrm{B}} T\left(w_{1} \delta n_{1}+w_{2} \delta n_{2}\right)=0,
$$

where $C \kappa^{2}=\partial^{2} f / \partial \psi^{2}$ with $\kappa$ being the inverse correlation length. Notice that if the ion densities are localized in regions shorter than $\xi=\kappa^{-1}, \delta \psi$ has Ornstein-Zernike tails in the form of Eq. (2.30) around such localized regions. Thus $w_{K}$ is written as

$$
w_{K}=-\left(4 \pi C / k_{\mathrm{B}} T\right) B_{K} \quad(K=1,2),
$$

where $B_{K}$ is the coefficient of the Ornstein-Zernike tail around an ion of the species $K$. If the two components of the fluid mixture have the same molecular size $a$ as assumed in the preceding section, $B_{K}$ is determined by the parameters $A_{K}$ in Eq. (2.22), where

$$
A_{1} / Z^{2}=A_{2}=\varepsilon_{1} \ell_{\mathrm{B}} / 8 \pi \varepsilon_{\mathrm{c}} a .
$$

In particular, in the linear solvation regime with small $A_{K}$, Eq. (2.36) yields

$$
w_{K}=-4 \pi A_{K} a / R_{\mathrm{B} K} \quad \text { (linear), }
$$


where $R_{\mathrm{B} K}$ is the Born radius of the ion species $K$. See Fig. 4 for the nonlinear behavior of $B_{K}$. That is, $B_{K} / a \sim A_{K}^{0.4}$ for large $A_{K}$ and $w_{K}$ can well exceed 1 .

In Ref. $16 w_{K}$ was related to the Born solvation free energy $\Delta G_{\mathrm{B} K}=-\left(Q_{K}^{2} / 2 R_{\mathrm{B} K}\right)(1-1 / \varepsilon)$ in Eq. (1.1) which should be defined for the two species. That is, treating $\varepsilon$ as a function of $\psi$, we obtain

$$
k_{\mathrm{B}} T w_{K}=\frac{\partial}{\partial \psi} \Delta G_{\mathrm{B} K}=-\varepsilon_{1} Q_{K}^{2} / 2 \varepsilon_{\mathrm{c}}^{2} R_{\mathrm{B} K},
$$

where the differentiation is taken at $\phi=\phi_{\mathrm{c}}$. Obviously, Eqs. (3.12) and (3.13) are equivalent from Eq. (2.22). This simple derivation cannot be used in the strong solvation regime, however.

\section{B. Fluctuations in one-phase states}

We consider small plane-wave fluctuations in a onephase state. The fluctuation contributions to $F$ in the bilinear order are written as

$$
\begin{aligned}
\delta F= & \int_{\mathbf{q}}\left[\frac{1}{2}\left(r+C q^{2}\right)\left|\psi_{\mathbf{q}}\right|^{2}+\frac{2 \pi}{\varepsilon_{\mathrm{c}} q^{2}}\left|\rho_{\mathbf{q}}\right|^{2}\right. \\
& \left.+k_{\mathrm{B}} T \sum_{K=1,2}\left(\frac{\left|n_{K \mathbf{q}}\right|^{2}}{2\left\langle n_{K}\right\rangle}+w_{K} n_{K \mathbf{q}} \psi_{\mathbf{q}}^{*}\right)\right],
\end{aligned}
$$

where $\int_{\mathbf{q}} \cdots=(2 \pi)^{-3} \int d \mathbf{q} \cdots$ denotes the integration over the wave vector $\mathbf{q}$ and

$$
r=\partial^{2} f / \partial \psi^{2} \text {. }
$$

The $\psi_{\mathbf{q}}, n_{K \mathbf{q}}$, and $\rho_{\mathbf{q}}$ are the Fourier transformations of $\delta \psi$, $\delta n_{K}$, and $\rho$, respectively. We may set

$$
r=a_{0}\left(T-T_{\mathrm{c}}\right)
$$

at the critical concentration, where the coefficient $a_{0}$ is of order $k_{\mathrm{B}} / a^{3}$ and is equal to $4 k_{\mathrm{B}} D_{1} / a^{3}$ with $D_{1}$ being defined by Eq. (2.26). In the mean field expression $\xi=\xi_{0+}\left(T / T_{\mathrm{c}}\right.$ $-1)^{-1 / 2}$ of the correlation length, we have

$$
a_{0}=C / \xi_{0+}^{2} T_{\mathrm{c}} \text {. }
$$

We may then calculate the structure factor for the order parameter $S(q)=\left\langle\left|\Psi_{\mathbf{q}}\right|^{2}\right\rangle$ in the mean field theory. Since the equilibrium distribution is given by const $\times \exp \left(-\delta F / k_{\mathrm{B}} T\right)$, the inverse of $S(q)$ is written as ${ }^{16,30}$

$$
\begin{aligned}
k_{\mathrm{B}} T / S(q)= & a_{0}\left(T-T_{\mathrm{c}}-\Delta T_{\text {ion }}\right) \\
& +C q^{2}\left[1-\gamma_{\mathrm{p}}^{2} /\left(1+\lambda_{\mathrm{D}}^{2} q^{2}\right)\right],
\end{aligned}
$$

where $\Delta T_{\text {ion }}$ is the critical temperature shift due to salt given by

$$
\Delta T_{\text {ion }}=\left(k_{\mathrm{B}} T_{\mathrm{c}} / a_{0}\right)\left(w_{1}+Z w_{2}\right)^{2} n_{\mathrm{tot}} /(1+Z)^{2},
$$

and $\lambda_{\mathrm{D}}$ is the Debye screening length defined by

$$
\lambda_{\mathrm{D}}^{-1}=\left(4 \pi Z n_{\mathrm{tot}} e^{2} / \varepsilon_{\mathrm{c}} k_{\mathrm{B}} T\right)^{1 / 2} .
$$

We introduce a dimensionless parameter,

$$
\begin{aligned}
\gamma_{\mathrm{p}} & =\left|w_{1}-w_{2}\right|\left(k_{\mathrm{B}} T / 4 \pi C \ell_{\mathrm{B}}\right)^{1 / 2} /(1+Z) \\
& =\left|B_{1}-B_{2}\right|\left(4 \pi C / \ell_{\mathrm{B}} k_{\mathrm{B}} T\right)^{1 / 2} /(1+Z) .
\end{aligned}
$$

This number is independent of the ion density and represents the strength of asymmetry in the ion-induced polarization between the two components. In the linear solvation regime with small $A_{K}$, we use Eq. (3.12) to obtain

$$
\gamma_{\mathrm{p}}=\frac{\varepsilon_{1} \sqrt{\ell_{\mathrm{B}} k_{\mathrm{B}} T}}{2 \varepsilon_{\mathrm{c}}(1+Z) \sqrt{4 \pi C}}\left|\frac{Z^{2}}{R_{\mathrm{B} 1}}-\frac{1}{R_{\mathrm{B} 2}}\right| \quad \text { (linear). }
$$

The structure factor of the charge density fluctuations $S_{\rho \rho}(q)=\left\langle\left|\rho_{\mathbf{q}}\right|^{2}\right\rangle / e^{2}$ is written as

$$
\begin{aligned}
S_{\rho \rho}(q)= & Z n_{\text {tot }} \frac{\lambda_{\mathrm{D}}^{2} q^{2}}{1+\lambda_{\mathrm{D}}^{2} q^{2}}+\left(w_{1}-w_{2}\right)^{2}\left(\frac{Z n_{\mathrm{tot}}}{Z+1}\right)^{2} \\
& \times\left(\frac{\lambda_{\mathrm{D}}^{2} q^{2}}{1+\lambda_{\mathrm{D}}^{2} q^{2}}\right)^{2} S(q),
\end{aligned}
$$

where the first term is the Debye-Hückel structure factor and the second term arises from the coupling to the order parameter.

From the structure factor $S(q)$ in Eq. (3.18) we may draw the following conclusions:

(i) If $\gamma_{\mathrm{p}}<1, S(q)$ is maximum at $q=0$ and the critical temperature shift due to ions is given by Eq. (3.19). As a rough estimate for the monovalent case $Z=1$, we set $a_{0}^{-1} \bar{n}$ $\sim k_{\mathrm{B}} T \xi_{+0}^{3} \bar{n} \sim c$, where $c$ is the mass or mole fraction of the ions. Then $\Delta T_{\text {ion }} \sim\left(w_{1}+w_{2}\right)^{2} c$. If $\left|w_{1}+w_{2}\right| \sim 3$, this result is consistent with the experiments. ${ }^{22,23}$ In future experiments, let $k_{\mathrm{B}} T / C S(q)$ versus $q^{2}$ be plotted; then, the slope is 1 $-\gamma_{\mathrm{p}}^{2}$ for $q \lambda_{\mathrm{D}} \ll 1$ and is 1 for $q \lambda_{\mathrm{D}} \gg 1$. This changeover should be detectable unless $\gamma_{\mathrm{p}} \ll 1$.

(ii) The case $\gamma_{\mathrm{p}}=1$ corresponds to a Lifshitz point, ${ }^{32,33}$ where $1 / S(q)-1 / S(0) \propto q^{4} /\left(1+q^{2} \lambda_{\mathrm{D}}^{2}\right)$.

(iii) If $\gamma_{\mathrm{p}}>1$, the structure factor attains a maximum at an intermediate wave number $q_{\mathrm{m}}$ given by

$$
q_{\mathrm{m}}=\left(\gamma_{\mathrm{p}}-1\right)^{1 / 2} / \lambda_{\mathrm{D}},
$$

so $q_{\mathrm{m}} \propto n_{\mathrm{tot}}^{1 / 2}$. The maximum of the structure factor $S\left(q_{\mathrm{m}}\right)$ is written as

$$
S\left(q_{\mathrm{m}}\right)=k_{\mathrm{B}} T /\left[a_{0}\left(T-T_{\mathrm{c}}-\Delta T_{\mathrm{ion}}^{\prime}\right)\right],
$$

where

$$
\Delta T_{\text {ion }}^{\prime}=\Delta T_{\text {ion }}+\left(\gamma_{\mathrm{p}}-1\right)^{2} T_{\mathrm{c}} \xi_{0+}^{2} / \lambda_{\mathrm{D}}^{2},
$$

where use has been made of Eq. (3.17). A charge-densitywave phase should be realized for $T-T_{c}<\Delta T_{\text {ion }}^{\prime}$. It is remarkable that this mesoscopic phase appears however small $n_{\text {tot }}$ is as long as $\gamma_{\mathrm{p}}>1$ and $q_{\mathrm{m}} L \gg 1$ with $L$ being the system length. Here relevant is the coupling of the order parameter and the charge density in the form $\propto \psi \rho$ in the free-energy density, which generally exists in ionic systems. This possibility of a mesoscopic phase was first predicted for electrolytes by Nabutovskii et al., ${ }^{30}$ but has not yet been confirmed in experiments. In polyelectrolytes, on the other hand, electric charges are attached to polymers and the structure factor of the polymer is known to take a form similar to Eq. (3.18), leading to a mesoscopic phase at low temperatures, in the Debye-Hückel approximation. ${ }^{32}$ 


\section{SUMMARY AND CONCLUDING REMARKS}

We summarize our results:

(i) In the first part, we have examined the concentration profile around a charged particle in a near-critical polar binary mixture, as can be seen in Figs. 1 and 2. We have started with the simple Ginzburg-Landau free energy (2.3) supplemented with the relations (2.1) and (2.2) of electrostatics. Preferential solvation can occur in the presence of a concentration-dependent dielectric constant and becomes strong when the parameter $A$ defined by Eq. (2.22) satisfies the condition (2.24). As the critical point is approached, the concentration has a long-range Ornstein-Zernike tail (2.30) in an intermediate range $a<r<\lambda$ as demonstrated in Fig. 3. We plot the coefficient $B$ versus $A$ in Fig. 4 and the crossover length $\lambda$ versus $A$ in Fig. 5. At the critical point $\psi(r)$ is long ranged in the form of Eq. (2.43) as demonstrated in Fig. 6. The solvation free energy $F_{\text {sol }}$ behaves as a function of $A$ as in Fig. 7.

(ii) In the second part of this work we have presented the coarse-grained free energy as in Eq. (3.4) where the order parameter and the ion densities are strongly coupled in the bilinear form. We have related the coupling constants $w_{1}$ and $w_{2}$ in terms of $B_{1}$ and $B_{2}$, the coefficients of the OrnsteinZernike tails for the ion, 1 and 2, respectively. Furthermore, if this coupling is significantly different between the two species of the ions, a charge-density-wave phase can be realized.

We then give some remarks.

(i) We have neglected the dielectric saturation near the ion. ${ }^{9,10}$ If this effect is taken into account, the growth of the coefficient $B$ with increasing $A$ would be weaker than in Fig. 4.

(ii) In future experiments, the structure factor (3.18) should be observed. There could be the case $\gamma_{\mathrm{p}}>1$, where the mesoscopic phase is formed at low temperatures, for pairs of small cations such as $\mathrm{Li}^{+}$or $\mathrm{Al}^{3+}$ and relatively large anions. ${ }^{1}$

(iii) Dynamical problems remain largely unsolved. For example, we are interested in the response of ions against ac electric field in near-critical polar binary mixtures. In the strong solvation condition the ion mobility should strongly depend on $\omega \tau_{\xi}$ where $\omega$ is the frequency of the field and $\tau_{\xi}$ is the order parameter lifetime $\left(=6 \pi \eta \xi^{3} / k_{\mathrm{B}} T\right.$ with $\eta$ being the shear viscosity ${ }^{15}$ ). Furthermore, even if $\omega \tau_{\xi} \ll 1$, the large-scale solvation cloud on the scale of $\xi$ should become nonlinearly dependent on the applied field.

(iv) Solvation or polarization effects due to inhomogeneous dielectric constant (or dielectric tensor for liquid crystals) can strongly influence the phase transition behavior in complex fluids including polyelectrolytes, charged gels, and liquid crystals containing ions or charged colloids. ${ }^{17}$ The importance of this effect in polyelectrolytes has recently been pointed out by Kramarenko et al..$^{34}$

(v) A similar Ginzburg-Landau theory can easily be developed on solvation effects in polar one-component fluids. For example, we may start with the free-energy density in the van der Waals theory ${ }^{15}$ in place of the Hildebrand freeenergy density (2.4). As regards statics salient features in one-component fluids are nearly the same as those in polar binary mixtures, but dynamics of critical electrostriction should be very different between the two cases. ${ }^{15}$

Note added in proof. The long-distance behavior (2.43) was also obtained in a different context of critical absorption by Hanke and Dietrich [Phys. Rev. E 59, 5801 (1999)].

\section{ACKNOWLEDGMENTS}

One of the authors (A.O.) would like to thank K. Orzechowski, M. Misawa, and M. Anisimov for valuable discussions on the ion effects in near-critical fluids. This work was supported by Grants in Aid for Scientific Research and for the 21st Centure COE "Center for Diversity and University in Physics" from the Ministry of Education, Culture, Sports, Science and Technology of Japan.

\section{APPENDIX: DERIVATION OF EQ. (2.17)}

In equilibrium $\phi(r)$ around a charged particle is determined by

$$
\hat{f}^{\prime}-C \nabla^{2} \phi-\frac{\varepsilon_{1}}{8 \pi \varepsilon^{2} r^{4}} Z^{2} e^{2} \theta^{2}=0,
$$

where $\hat{f}$ is defined in Eq. (2.16). Multiplying $\phi^{\prime}=d \phi / d r$ and integrating over $r$ in the range $[r, \infty]$, we obtain

$$
\hat{f}+\frac{Z^{2} e^{2}}{8 \pi \varepsilon r^{4}} \theta^{2}-\frac{C}{2}\left(\phi^{\prime}\right)^{2}=-\int_{r}^{\infty} d r_{1} H\left(r_{1}\right),
$$

where

$$
H(r)=\frac{2 C}{r}\left(\phi^{\prime}\right)^{2}+\frac{Z^{2} e^{2}}{8 \pi \varepsilon} \frac{d}{d r}\left(\frac{\theta^{2}}{r^{4}}\right) .
$$

Integration of Eq. (A2) over the whole space yields Eq. (2.17) with the aid of $\int_{0}^{\infty} d r r^{2} \int_{r}^{\infty} d r_{1} H\left(r_{1}\right)=\int_{0}^{\infty} d r r^{3} H(r) / 3$.

${ }^{1}$ J. N. Israelachvili, Intermolecular and Surface Forces (Academic London, 1991).

${ }^{2}$ Y. Marcus, Ion Solvation (Wiley, New York, 1985).

${ }^{3}$ J. Tomasi and M. Persico, Chem. Rev. (Washington, D.C.) 94, 2027 (1994)

${ }^{4}$ O. Kajimoto, Chem. Rev. (Washington, D.C.) 99, 355 (1999).

${ }^{5}$ C. J. Cramer and D. G. Truhlar, Chem. Rev. (Washington, D.C.) 99, 2161 (1999).

${ }^{6}$ M. Orozco and F. J. Luque, Chem. Rev. (Washington, D.C.) 100, 4187 (2000)

${ }^{7}$ M. Born, Z. Phys. 1, 45 (1920).

${ }^{8}$ Y. Marcus, Chem. Rev. (Washington, D.C.) 88, 1475 (1988).

${ }^{9}$ M. H. Abraham, J. Liszi, and L. Meszaros, J. Chem. Phys. 70, 2491 (1979).

${ }^{10}$ L. Sandberg and O. Edholm, J. Chem. Phys. 116, 2936 (2002).

${ }^{11}$ J. Padova, J. Phys. Chem. 72, 796 (1968).

${ }^{12}$ R. H. Wood, J. R. Qulnt, and J.-P. E. Grolier, J. Phys. Chem. 85, 3944 (1981).

${ }^{13}$ H. Luo and S. C. Tucker, J. Phys. Chem. 100, 11165 (1996).

${ }^{14}$ G. Zimmerli, R. A. Wilkinson, R. A. Ferrell, and M. R. Moldover, Phys. Rev. E 59, 5862 (1999). Here critical electrostriction was measured optically around a wire conductor in $\mathrm{SF}_{6}$.

${ }^{15}$ A. Onuki, Phase Transition Dynamics (Cambridge University Press, Cambridge, 2002).

${ }^{16}$ A. Onuki, in Nonlinear Dielectric Phenomena in Complex Liquids, NATO Advanced Studies Institute, Series II: Vol. 157, edited by S. J. Rzoska (Kluwer Academic, Dordrecht, 2004).

${ }^{17}$ A. Onuki, J. Phys. Soc. Jpn. 73, 511 (2004).

${ }^{18}$ P. Debye and K. Kleboth, J. Chem. Phys. 42, 3155 (1965). If the Landau expansion of the free energy density is written as $f=k_{B}\left(T-T_{c}\right) \psi^{2} / 2 b_{0}^{3}$ 
$+\cdots$, their data suggest $b_{0}=4.3 \AA$. In our theory we have $b_{0}$ $=a /\left(2 D_{1}\right)^{1 / 3}$. We expect that $D_{1}$ is of order 1 and $a \sim \xi_{0+} \sim b_{0}$.

${ }^{19}$ J. H. Hildebrand and R. L. Scott, Regular Solutions (Prentice-Hall, Englewood Cliffs, NJ, 1962).

${ }^{20}$ S. A. Safran, Statistical Thermodynamics of Surfaces, Interfaces, and Membranes (Westview, Boulder, CO, 2003).

${ }^{21}$ If we set $C=2 \alpha k_{\mathrm{B}} T / a$, our results near the critical point are those at $\alpha$ $=1$. Obviously, results for general $\alpha$ can be obtained from ours by simple scale changes. For example, Fig. 4 becomes the relation of $B \alpha^{1 / 2} / a$ versus $A \alpha^{2}$.

${ }^{22}$ E. L. Eckfeldt and W. W. Lucasse, J. Phys. Chem. 47, 164 (1943).

${ }^{23}$ B. J. Hales, G. L. Bertrand, and L. G. Hepler, J. Phys. Chem. 70, 3970 (1966).

${ }^{24}$ V. Balevicius and H. Fuess, Phys. Chem. Chem. Phys. 1, 1507 (1999).

${ }^{25}$ J. L. Tveekrem and D. T. Jacobs, Phys. Rev. A 27, 2773 (1983).

${ }^{26}$ T. Narayanan and A. Kumar, Phys. Rep. 249, 135 (1994).

${ }^{27}$ J. Jacob, A. Kumar, S. Asokan, D. Sen, R. Chitra, and S. Mazumder, Chem. Phys. Lett. 304, 180 (1999).
${ }^{28}$ J. Jacobs, A. Kumar, M. A. Anisimov, A. A. Povodyrev, and J. V. Sengers, Phys. Rev. E 58, 2188 (1998); M. A. Anisimov, J. Jacob, A. Kumar, V. A. Agayan, and J. V. Sengers, Phys. Rev. Lett. 85, 2336 (2000).

${ }^{29}$ M. Misawa, K. Yoshida, K. Maruyama, H. Munemura, and Y. Hosokawa, J. Phys. Chem. Solids 60, 1301 (1999); K. Yoshida, M. Misawa, K. Maruyama, M. Imai, and M. Furusaka, J. Chem. Phys. 113, 2343 (2000).

${ }^{30}$ V. M. Nabutovskii, N. A. Nemov, and Yu. G. Peisakhovich, Phys. Lett. 79A, 98 (1980); Sov. Phys. JETP 52, 111 (1980) [Zh. Eksp. Teor. Fiz. 79, 2196 (1980)]; Mol. Phys. 54, 979 (1985).

${ }^{31}$ V. L. Koulinskii, N. P. Malomuzh, and V. A. Tolpekin, Phys. Rev. E 60, 6897 (1999).

${ }^{32}$ V. Yu. Boryu and I. Ya. Erukhimovich, Macromolecules 21, 3240 (1988); J. F. Joanny and L. Leibler, J. Phys. (France) 51, 545 (1990).

${ }^{33}$ P. M. Chaikin and T. C. Lubensky, Principles of Condensed Matter Physics (Cambridge University Press, Cambridge, 1995).

${ }^{34}$ Yu. Kramarenko, I. Ya. Erukhimovich, and A. R. Khokhlov, Macromol. Theory Simul. 11, 462 (2002). 